\title{
Engagement of General Practice in an Australian Organised Bowel Cancer Screening Program: A Cross-Sectional Survey of Knowledge and Practice
}

\author{
Carol A Holden ${ }^{1 *}$, Oliver Frank ${ }^{2}$, Ming Li ${ }^{3}$, Ramesh Manocha ${ }^{4}$, Joanna Caruso ${ }^{1}$, \\ Deborah Turnbull ${ }^{5}$, Richard L Reed ${ }^{6}$, Caroline L Miller ${ }^{1,7}$, David Roder ${ }^{3}$, \\ Ian Olver ${ }^{5}$
}

\begin{abstract}
Background: Understanding factors causing variation in family physicians/general practitioners (GPs) screening knowledge, understanding and support of organised population-based colorectal cancer (CRC) programs can direct interventions that maximise the influence of a CRC screening recommendation from a GP. This study aims to assess contextual factors that influence knowledge and quality improvement (QI) practice directed to CRC screening in Australian general practice. Methods: A convenience sample of anonymous general practice staff from all Australian states and territories completed a web-based survey. Multivariate analyses assessed the association between CRC screening knowledge and QI-CRC practice scores and patient, organisational and environmental-level contextual factors. Results: Of 1,013 survey starts, 918 respondents $(90.6 \%)$ completed the survey. Respondents less likely to recommend FOBT screening had lower knowledge and QI practice scores directed to CRC screening. Controlling for individual and practice characteristics, respondents' rating of the Australian National Bowel Cancer Screening Program (NBCSP) support for preventive care, attending external education, and sufficient practice resources to implement QI practice (generally) were the strongest factors associated with QI practice directed towards CRC screening. Knowledge scores were less amenable to the influence of contextual factors explored. Conclusion: More active engagement of family medicine/general practice to improve screening promotion could be achieved through better QI resourcing without changing the fundamental design of population-based CRC screening programs.
\end{abstract}

Keywords: Colorectal cancer- mass screening- primary health care- quality improvement- quantitative

Asian Pac J Cancer Prev, 21 (7), 2099-2107

\section{Introduction}

Population-based colorectal cancer (CRC) screening programs worldwide aim to reduce CRC mortality through early disease detection (Navarro et al., 2017). In Australia, the National Bowel Cancer Screening Program (NBCSP) was introduced incrementally from 2006, to be complete in 2020, when all Australians aged 50-74 years will receive immunochemical faecal occult blood test (FOBT) kits directly to their home every two years (National Bowel Cancer Screening Program, 2016; Australian Institute of Health and Welfare, 2019). However, 15-years on, the Australian participation rate remains suboptimal at $41 \%$ (Australian Institute of Health and Welfare, 2019), partly due to the phased implementation process
(Flitcroft et al., 2010), and limited involvement of family physicians/general practitioners (GPs) (Grogan and Olver, 2014).

The NBCSP is administered nationally with family physicians/GPs indirectly expected to encourage participation, particularly for non-adherent patients, and follow-up and referral for diagnostic (colonoscopy) assessment (National Bowel Cancer Screening Program, 2016). A FOBT screening recommendation from a family physician/GP is the most influential factor in a patient's participation in CRC screening (Cole et al., 2002; Zajac et al., 2010). Yet, variation in practitioner screening knowledge and attitude appears to influence their CRC screening practice (Perin et al., 2015; Dawson et al., 2017; Şahin and Aker, 2017), although Australian studies

${ }^{1}$ South Australian Health and Medical Research Institute, Adelaide, Australia. ${ }^{2}$ Discipline of General Practice, Adelaide Medical School, University of Adelaide, Adelaide, and Oakden Medical Centre, Hillcrest, Australia. ${ }^{3}$ Cancer Epidemiology and Population Health, Cancer Research Institute, University of South Australia, Adelaide, Australia. ${ }^{4}$ Healthed Pty Ltd, New South Wales, Australia. ${ }^{5}$ School of Psychology, University of Adelaide, Adelaide, Australia. ${ }^{6}$ Discipline of General Practice, Flinders University, Adelaide, Australia. ${ }^{7}$ School of Public Health, University of Adelaide, Adelaide, Australia. *For Correspondence: carol.holden@ sahmri.com 
pre-date the NBCSP implementation (Youl et al., 2006).

Despite practitioner CRC screening surveys identifying individual and practice factors that moderate screening practice (National Cancer Institute (NCI); Damery et al., 2010; Perin et al., 2015; Şahin and Aker, 2017), and numerous systematic reviews (including meta-analyses) describing effective primary care interventions (Emery et al., 2014), there has been little research progress demonstrating sustained improvements in CRC screening participation in routine practice (Dodd et al., 2018). This may be related to limited recognition of the diverse contextual influencers that moderate the implementation of primary care interventions (Moore et al., 2015; Lau et al., 2016). Little is known about those contextual factors that may influence whether family physicians/GPs advise their eligible patients to be screened (Davis et al., 2018). We hypothesise that understanding those factors that influence practitioner CRC screening knowledge and practice will identify intervention points to increase FOBT screening recommendations. In the context of a broader quality improvement study, we surveyed Australian general practice to identify these influential factors. The findings will support a more coordinated effort to support the general practice workforce in improving CRC screening participation rates.

\section{Materials and Methods}

We undertook a cross-sectional web-based survey, informed by a recent systematic scoping review (Holden et al., 2020), to explore individual, practice and contextual factors that might influence screening recommendations in Australian general practice.

\section{Participants}

An opportunistic, convenience sample of general practice staff was surveyed using a sampling frame of practitioner email addresses, registered with an education provider (Healthed Pty Ltd), who consented to receive email communication from the provider. A chance to win one of five \$AUS100 gift vouchers was offered to those that completed the survey (Vangeest et al., 2007).

\section{Data collection}

Study data (predominately categorical responses and 5-point Likert scales) were collected and managed using REDCap (Research Electronic Data Capture), a secure, web-based application (Harris et al., 2009). A valid response was needed for survey completion with no constraints imposed on the response range for three knowledge-based and two open-response questions. The survey was open from 24 September to 10 October 2018 , with one reminder sent to non-respondents after 10 days. The 50-item survey took on average less than five minutes to complete.

\section{Survey instrument}

Survey questions (Supplementary Data 1) were sourced primarily from the US National Cancer Institute Survey of Colorectal Cancer Screening Practices, and modified by Damery and colleagues (UK) (Damery et al., 2010), and as relevant for the Australian context (Britt et al., 2015). Questions included knowledge of Australian CRC screening guidelines (Cancer Council Australia Colorectal Cancer Guidelines Working Party, 2017), quality improvement (QI) practices, both general and specifically directed to colorectal cancer (QI-CRC) (The Royal Australian College of General Practitioners (RACGP), 2016), screening participation and individual socio-demographic (e.g. age) and practice characteristics (e.g. practice size). Geographical setting (Australian Statistical Geography Standard (ASGS) remoteness structure) (Australian Bureau of Statistics (ABS), 2018) and relative socio-economic disadvantage (SocioEconomic Indexes for Areas, SEIFA) (Australian Bureau of Statistics (ABS), 2016) were generated from practice postcode.

Eight GPs from a large family/general practice initially tested the survey for design, content and completion time. A random sample of 18 GPs from metropolitan practices then pilot tested the survey for comprehension and response logic before final dissemination.

\section{Study outcome}

CRC screening 'knowledge', and 'QI practice' scores were calculated for each respondent based on the responses to three specific questions for each study outcome (Supplementary Data 2). Knowledge scores were dichotomised between those with a maximum possible knowledge score of nine and those scoring less (gaps in knowledge). QI-CRC screening practice score was dichotomised between those who were active in QI-CRC activity (score $=7-9$ ) and those scoring less (low-moderate QI-CRC activity, <7).

\section{Study factors}

FOBT screening recommendation and potential drivers (contextual factors) that might be associated with knowledge and QI practice directed to CRC screening recommendation were studied. Responses to 'how often do you recommend FOBT for your asymptomatic, average-risk patients' were dichotomised between 'always' and 'not always' (responses other than 'always').

A three-level (patient, organisation and environmental) complexity framework of contextual factors, modified from Lau et al. (Lau et al., 2016), was applied with selected factors identified from the literature (Holden et al., 2020):

i) patient-level factors (practice demographic profile (Davis et al., 2018)): respondents understanding of their patient profile for the targeted age-group (aged 50 years or older) and under-screened population subgroups (e.g. Aboriginal and/or Torres Strait Islander clients; low socio-economic status, SES).

ii) organisation/system-level factors (existing QI resources and activity (Arroyave et al., 2011)): for each respondent, a total QI activity score (range, three to nine) was calculated from their self-reported use of data extraction tools, measurement and discussion of practice performance data (Supplementary Data 2). QI scores were categorised into three groups reflective of their general QI-activity level: low QI activity (score $=3$ ); medium QI activity ( score $=4-6$ ); active QI (score $=7-9$ ). 
iii) environmental-level factors (infrastructure (i.e. NBCSP) (Tong et al., 2004), financial incentives (Mauro et al., 2019) and external education activities): respondents rated the extent that the NBCSP improves care (Unsatisfactory, <40; Neutral, 40-60; Significantly improves care, $>60$ ), the influence of a financial incentive (Australian Government Practice Incentives Program, PIP) on CRC screening participation and attendance at quality care/prevention education activities in the past two years.

\section{Statistical analyses}

The mean (and standard deviation) knowledge and QI-CRC practice score was presented and compared by respondent sociodemographic and practice characteristics and study factors measured using parametric or non-parametric tests as appropriate.

The association (OR) between knowledge and QI-CRC practice and the study factors measured was assessed using logistic regression analysis. The adjusted association was from models including respondents' sociodemographic and practice characteristics. In addition, a step-wise logistic regression analysis was conducted by including all measured study factors and covariates to identify factors significantly associated with knowledge and QI-CRC practice.

All analyses were conducted using STATA 14 (STATACorp, College Station, Texas, USA). Results were considered statistically significant at $\mathrm{p}<0.05$.

\section{Ethics approval}

This survey was part of a larger study approved by the University of South Australia Human Research Ethics Committee (Application ID: 201061). Participants choosing to complete the online survey were deemed as providing consent, as approved by the ethics committee.

\section{Results}

Of the 1,013 recipients who started the survey, 918 surveys were available for analysis. According to the American Association for Public Opinion Research (AAPOR) (American Association for Public Opinion Research, 2016), this yields a Cooperation Rate 4 (COOP4), which includes partial responses, of 90.6\%. There were no significant differences between the 'Incomplete' and 'Complete' survey respondents in general practice characteristics (Supplementary Data 3).

Respondent, individual and practice characteristics are presented in Table 1. Most respondents were female, aged over 55 years, completed their medical training in Australia and had personal experience of CRC screening: for those who were screened, $40 \%(n=218)$ were screened via FOBT only, 30\% $(\mathrm{n}=166)$ via FOBT and colonoscopy, and $30 \%(n=164)$ via colonoscopy only. Respondents worked in practices from all states and territories, with most working in practices in major cities and a gradient in the socio-economic practice setting. Most respondents knew their practice demographic relating to age and socio-economic status, however fewer knew their practice proportion of under-screened populations (e.g. Aboriginal and Torres Strait Islander clients, non-English speaking patients and new migrants/refugees).

Respondent characteristics were different from the Australian general practice workforce (Commonwealth Department of Health) (Supplementary Data 4). When compared to other Australian studies where a small proportion of general practice respondents were drawn from a larger workforce sampling frame (e.g. BEACH data (Britt et al., 2015)), respondents were more likely to be female $\left(\chi^{2} 247.85, \mathrm{p}<0.001\right)$ and from different states $\left(\chi^{2} 19.24, \mathrm{p}=0.01\right)$ (Supplementary Data 4$)$.

\section{Colorectal cancer (CRC) screening knowledge and} QI-CRC practice

Mean knowledge scores about CRC screening were higher for females, younger respondents (both in years of practice and in age), those who had not previously been screened and respondents from group practices (Table 1). QI practices relating to CRC screening (QI-CRC) were not different by practice characteristics, however male respondents, Australian-trained respondents and those with fewer years of practice had significantly better QI-CRC practice scores than their respective counterparts (Table 1).

\section{FOBT screening recommendation and knowledge and QI-CRC practice}

Half of respondents 'always' recommended FOBT to their eligible patients $(n=424,50 \%)$. About a third of respondents always/very often recommended colonoscopy $(n=308,36 \%)$ despite no Australian guidelines recommending these tests as screening procedures for asymptomatic, average-risk patients.

Those respondents who 'always' recommended FOBT to their asymptomatic, average-risk patients had higher mean scores for knowledge (8.2 ( $\mathrm{SD} \pm 0.8)$ vs 8.0 (SD $\pm 1.0), \mathrm{p}<0.013)$ and QI-CRC practice (6.3 ( $\mathrm{SD} \pm 2.0) \mathrm{vs}$ $5.6(\mathrm{SD} \pm 1.9), \mathrm{p}<0.001)$, than those who did not 'always' recommend FOBT screening to their eligible patients.

Contextual factors and their association with CRC screening knowledge and QI-CRC practice

Lower knowledge scores were observed in practices not servicing the target population ( $>50$ years) (Table 2 ). Servicing under-screened population groups did not appear to influence CRC screening knowledge scores (Table 2 and Table 3). Similarly, none of the organisation-level or environmental-level factors explored influenced knowledge scores (Table 2 and Table 3), except that a financial incentive would not make a difference to CRC screening practice for those respondents with higher knowledge scores (Table 3).

In contrast, while patient-level factors did not appear to influence QI-CRC practice scores, organisational and environmental-level factors had varying influence on active QI-CRC practice. Those respondents who reported having insufficient resources to implement QI programs, reported lower QI-CRC practice scores (Table 2) after adjusting for individual and practice characteristics (Table 3). Similarly, environmental-level factors had a positive influence on QI-CRC practice: respondents who 
Table 1. Knowledge and Quality Improvement Practice Scores Directed to Bowel Cancer Screening by Respondent Socio-Demographic and Practice Characteristics

\begin{tabular}{|c|c|c|c|c|c|}
\hline \multirow{2}{*}{$\begin{array}{l}\text { Socio-demographic characteristic } \\
\text { Gender }\end{array}$} & \multirow[t]{2}{*}{ Number (\%) } & \multicolumn{2}{|c|}{ Knowledge Mean Score (SD) } & \multicolumn{2}{|c|}{ QI-CRC Practice Mean Score (SD) } \\
\hline & & & $\mathrm{p}=0.003$ & & $\mathrm{p}=0.009$ \\
\hline Male & $171(20 \%)$ & $7.9(0.9)$ & & $6.3(2.0)$ & \\
\hline Female & $647(79 \%)$ & $8.1(0.9)$ & & $5.8(2.0)$ & \\
\hline Invalid/prefer not to say & $4(1 \%)$ & $7.9(0.9)$ & & $4.8(2.9)$ & \\
\hline Age & & & $\mathrm{p}<0.001$ & & $\mathrm{p}=0.704$ \\
\hline$<35$ years & $74(9 \%)$ & $8.4(1.0)$ & & $6.1(2.0)$ & \\
\hline $35-44$ years & $147(18 \%)$ & $8.4(0.7)$ & & $5.9(1.9)$ & \\
\hline $45-54$ years & $192(24 \%)$ & $7.9(0.9)$ & & $5.8(2.1)$ & \\
\hline $55+$ years & $403(49 \%)$ & $7.9(0.9)$ & & $6.0(2.0)$ & \\
\hline Country of Graduation & & & $\mathrm{p}=0.878$ & & $\mathrm{p}<0.001$ \\
\hline Australia & $530(70 \%)$ & $8.1(0.9)$ & & $5.7(1.9)$ & \\
\hline Overseas & $222(30 \%)$ & $8.1(0.8)$ & & $6.5(2.0)$ & \\
\hline Years of practice (years) & & & $\mathrm{p}<0.001$ & & $\mathrm{p}=0.057$ \\
\hline$<5$ & $153(19 \%)$ & $8.4(0.9)$ & & $6.2(2.0)$ & \\
\hline $6-10$ & $130(16 \%)$ & $8.2(0.8)$ & & $6.0(1.9)$ & \\
\hline $11-19$ & $109(13 \%)$ & $8.2(0.8)$ & & $6.1(2.1)$ & \\
\hline $20+$ & $428(52 \%)$ & $7.9(0.9)$ & & $5.7(2.0)$ & \\
\hline Personally screened & & & $\mathrm{p}<0.001$ & & $\mathrm{p}=0.618$ \\
\hline Screened & $549(65 \%)$ & $8.0(0.9)$ & & $5.9(2.0)$ & \\
\hline Not screened & $283(33 \%)$ & $8.3(0.8)$ & & $5.9(2.0)$ & \\
\hline Prefer not to say & $18(2 \%)$ & $7.9(0.7)$ & & $6.4(1.9)$ & \\
\hline Practice characteristic & Number $(\%)$ & Knowledge & Score (SD) & QI-CRC Prac & in Score (SD) \\
\hline Nos. GPs working at practice & & & $\mathrm{p}=0.002$ & & $\mathrm{p}=0.330$ \\
\hline Sole provider & $45(5 \%)$ & $7.6(1.2)$ & & $6.2(2.1)$ & \\
\hline $2-3$ & $165(20 \%)$ & $8.0(0.9)$ & & $6.1(2.1)$ & \\
\hline $4-9$ & $444(53 \%)$ & $8.2(0.8)$ & & $5.9(2.0)$ & \\
\hline $10+$ & $190(23 \%)$ & $8.2(0.8)$ & & $5.8(1.9)$ & \\
\hline Nos. nursing staff (FTE) & & & $\mathrm{p}=0.460$ & & $\mathrm{p}=0.414$ \\
\hline Nil & $79(9 \%)$ & $7.9(1.0)$ & & $5.6(2.1)$ & \\
\hline$<1.0$ & $287(34 \%)$ & $8.2(0.8)$ & & $6.0(1.9)$ & \\
\hline $1.0-3.0$ & $350(41 \%)$ & $8.1(0.8)$ & & $5.9(2.0)$ & \\
\hline $3.1-6.0$ & $110(13 \%)$ & $8.0(0.9)$ & & $6.2(2.0)$ & \\
\hline $6.1+$ & $22(3 \%)$ & $8.2(0.9)$ & & $5.9(2.1)$ & \\
\hline Practice ownership model & & & $\mathrm{p}=0.011$ & & $\mathrm{p}=0.427$ \\
\hline GP-owned & $591(70 \%)$ & $8.1(0.8)$ & & $5.9(1.9)$ & \\
\hline Corporate practice & $138(16 \%)$ & $8.1(0.9)$ & & $5.9(1.9)$ & \\
\hline Community-controlled & $53(6 \%)$ & $8.1(1.0)$ & & $6.0(1.9)$ & \\
\hline Other & $67(8 \%)$ & $7.7(1.1)$ & & $5.5(2.1)$ & \\
\hline \multicolumn{2}{|c|}{ Relative Socio-economic disadvantage index quintile } & & $\mathrm{p}=0.720$ & & $\mathrm{p}=0.214$ \\
\hline 1st (most disadvantage) & $98(12 \%)$ & $8.0(0.9)$ & & $6.0(2.1)$ & \\
\hline $2-5$ & $147(18 \%)$ & $8.0(0.9)$ & & $6.0(2.1)$ & \\
\hline $6-10$ & $152(18 \%)$ & $8.1(0.8)$ & & $6.1(1.9)$ & \\
\hline $11-19$ & $148(18 \%)$ & $8.1(0.9)$ & & $5.7(2.0)$ & \\
\hline 5th (least disadvantage) & $294(35 \%)$ & $8.1(0.9)$ & & $5.8(1.9)$ & \\
\hline \multicolumn{2}{|c|}{ ASGS - Remoteness Assessment (practice setting) } & & $\mathrm{p}=0.525$ & & $\mathrm{p}=0.232$ \\
\hline Major cities & $617(74 \%)$ & $8.1(0.9)$ & & $5.9(2.0)$ & \\
\hline Inner regional & $146(17 \%)$ & $8.1(0.8)$ & & $6.2(2.0)$ & \\
\hline Outer regional or remote & $76(9 \%)$ & $8.2(0.9)$ & & $5.9(2.1)$ & \\
\hline
\end{tabular}


positively rated the NBCSP as effectively supporting preventive care, and those respondents who recently completed external education, reported significantly higher QI-CRC practice scores (Table 2) and these activities increased the likelihood of active QI-CRC practice (Table 3). A small proportion of respondents with lower QI-CRC practice scores indicated that a financial incentive would make a difference to their CRC screening practice (Table 2) but this association did not persist when controlling for individual and practice characteristics (Table 3).

In the final full step-wise logistic regression model, factors significantly associated with higher CRC screening knowledge and QI-CRC practice score included younger respondents ( $<55$ years) who were $130 \%$ more likely $(95 \%$ CI 1.7-3.1), while those who indicated that a financial incentive would not influence their screening practice were $30 \%$ less likely (OR $0.7,95 \%$ CI $0.5-0.9$ ) to achieve full knowledge scores. Being active in QI-CRC was more common among male respondents (OR 1.7, 95\% CI $1.2-2.4$ ), those practicing for less than 20 years (OR 1.5, $95 \%$ CI 1.1-2.0) and respondents who positively rated the NBCSP(OR 1.5, 95\% CI 1.1-2.1), had sufficient resources (OR 2.1, 95\% CI 1.4-3.2), were actively implementing QI practice (OR 2.0, 95\% CI 1.4-3.2) and who had within the past two years attended external education activities (OR 1.7, 95\% CI 1.1-2.8).

Table 2. Knowledge and Quality Improvement Practice Scores Directed to Bowel Cancer Screening and Association with Selected Contextual Factors

\begin{tabular}{|c|c|c|c|c|c|}
\hline \multirow{2}{*}{$\begin{array}{l}\text { Contextual factors } \\
\text { Patient-level factors }\end{array}$} & \multirow[t]{2}{*}{ Number } & \multicolumn{2}{|c|}{ Knowledge Mean Score (SD) } & \multicolumn{2}{|c|}{ QI-CRC Practice Mean Score (SD) } \\
\hline & & & & & \\
\hline Demographic: $50+$ yr olds & & & $\mathrm{p}<0.001$ & & $\mathrm{p}=0.885$ \\
\hline None & $7(1 \%)$ & $6.3(1.3)$ & & $6.0(2.3)$ & \\
\hline Low-Moderate & $820(97 \%)$ & $8.1(0.9)$ & & $5.9(2.0)$ & \\
\hline I don't know & $20(2 \%)$ & $8.1(0.8)$ & & $6.2(1.8)$ & \\
\hline \multicolumn{2}{|c|}{ Demographic: Aboriginal and Torres Strait Islander } & & $\mathrm{p}=0.463$ & & $\mathrm{p}=0.060$ \\
\hline None & $413(49 \%)$ & $8.1(0.9)$ & & $5.8(2.0)$ & \\
\hline Low-Moderate & $236(28 \%)$ & $8.1(0.8)$ & & $6.1(2.1)$ & \\
\hline I don't know & $192(23 \%)$ & $8.0(0.9)$ & & $6.0(2.0)$ & \\
\hline Demographic: Low SES & & & $\mathrm{p}=0.031$ & & $\mathrm{p}=0.799$ \\
\hline None & $89(11 \%)$ & $7.9(1.1)$ & & $6.1(1.9)$ & \\
\hline Low-Moderate & $706(84 \%)$ & $8.1(0.8)$ & & $5.9(2.0)$ & \\
\hline I don't know & $50(6 \%)$ & $8.0(0.8)$ & & $5.8(1.9)$ & \\
\hline \multicolumn{6}{|l|}{ Organisational-level factors } \\
\hline Sufficient QI resources & & & $\mathrm{p}=0.903$ & & $\mathrm{p}<0.001$ \\
\hline Insufficient & $91(11 \%)$ & $8.1(0.8)$ & & $5.0(1.9)$ & \\
\hline Neutral & $177(21 \%)$ & $8.1(0.9)$ & & $5.3(1.8)$ & \\
\hline Sufficient & $585(69 \%)$ & $8.1(0.9)$ & & $6.3(2.0)$ & \\
\hline QI activity level & & & $\mathrm{p}=0.289$ & & $\mathrm{p}<0.001$ \\
\hline Low level QI & $302(35 \%)$ & $8.2(0.9)$ & & $5.1(1.7)$ & \\
\hline Medium QI & $330(39 \%)$ & $8.0(0.9)$ & & $5.9(1.9)$ & \\
\hline Active QI & $221(59 \%)$ & $8.0(0.9)$ & & $7.1(1.8)$ & \\
\hline \multicolumn{6}{|l|}{ Environmental-level factors } \\
\hline NBCSP Rating Score & & & $\mathrm{p}=0.387$ & & $\mathrm{p}<0.001$ \\
\hline Unsatisfactory & $90(11 \%)$ & $8.2(0.8)$ & & $4.4(1.8)$ & \\
\hline Meets expectations & $257(31 \%)$ & $8.0(1.0)$ & & $5.2(2.1)$ & \\
\hline Significantly improves care & $491(59 \%)$ & $8.1(0.9)$ & & $5.2(2.1)$ & \\
\hline PIP payment & & & $\mathrm{p}=0.130$ & & $\mathrm{p}=0.058$ \\
\hline Would make no difference & $593(71 \%)$ & $8.1(0.8)$ & & $6.0(2.0)$ & \\
\hline Neutral & $162(20 \%)$ & $8.1(1.0)$ & & $5.8(1.8)$ & \\
\hline Would make a difference & $75(9 \%)$ & $7.9(1.0)$ & & $5.5(2.1)$ & \\
\hline External education & & & $\mathrm{p}=0.67$ & & $\mathrm{p}=0.01$ \\
\hline Yes & $680(80 \%)$ & $8.1(0.9)$ & & $6.0(2.0)$ & \\
\hline No & $99(12 \%)$ & $8.1(0.8)$ & & $5.4(1.8)$ & \\
\hline I don't remember & $71(8 \%)$ & $8.0(1.1)$ & & $5.8(2.1)$ & \\
\hline
\end{tabular}


Table 3. Results of Logistic Regression Models Estimating the Association between Selected Contextual Factors and Full Knowledge Score* and Active QI Practice** for Bowel Cancer Screening in Australian General Practice

\begin{tabular}{|c|c|c|c|c|}
\hline & \multicolumn{2}{|c|}{ Full knowledge score* } & \multicolumn{2}{|c|}{ Active QI practice** } \\
\hline & Number $(\%)$ & Adjusted OR (95\% CI) & Number $(\%)$ & Adjusted OR $(95 \% \mathrm{CI})$ \\
\hline \multicolumn{5}{|l|}{ Demographic: $50+$ yr olds } \\
\hline None & 0 & NA & $3(0.8 \%)$ & $0.8(0.1-11.0)$ \\
\hline Low-Moderate & $319(97.9 \%)$ & Reference & $349(96.4 \%)$ & Reference \\
\hline I don't know & $7(2.2 \%)$ & $0.5(0.7-1.8)$ & $10(2.7 \%)$ & $1.6(0.5-5.2)$ \\
\hline \multicolumn{5}{|c|}{ Demographic: Aboriginal and Torres Strait Islander } \\
\hline None & $156(48.5 \%)$ & $1.0(0.7-1.6)$ & $163(45.7 \%)$ & $0.8(0.5-1.2)$ \\
\hline Low-Moderate & $97(30.1 \%)$ & Reference & $108(30.3 \%)$ & Reference \\
\hline I don't know & $69(21.4 \%)$ & $1.0(0.6-1.6)$ & $86(24.1 \%)$ & $1.1(0.7-1.7)$ \\
\hline \multicolumn{5}{|l|}{ Demographic: Low SES } \\
\hline None & $29(9 \%)$ & $0.7(0.4-1.3)$ & $43(11.9 \%)$ & $1.5(0.9-2.5)$ \\
\hline Low-Moderate & $281(87.0 \%)$ & Reference & $297(82.5 \%)$ & Reference \\
\hline I don't know & $13(4.0 \%)$ & $0.4(0.2-1.0)$ & $20(5.6 \%)$ & $0.9(0.4-1.8)$ \\
\hline \multicolumn{5}{|l|}{ Sufficient QI resources } \\
\hline Insufficient & $34(10.4 \%)$ & $1.1(0.6-2.0)$ & $20(5.5 \%)$ & $0.9(0.4-1.7)$ \\
\hline Neutral & $69(21.0 \%)$ & Reference & $47(13.0 \%)$ & Reference \\
\hline Sufficient & $225(68.6 \%)$ & $1.2(0.8-1.8)$ & $296(81.5 \%)$ & $3.0(2.0-4.6)$ \\
\hline \multicolumn{5}{|l|}{ QI practice } \\
\hline Low level QI & $122(37.2 \%)$ & $0.7(0.5-1.1)$ & $73(20.1 \%)$ & $0.1(0.1-0.2)$ \\
\hline Medium QI & $118(36.0 \%)$ & $0.8(0.5-1.2)$ & $148(40.8 \%)$ & $0.4(0.3-0.6)$ \\
\hline Active QI & $88(26.8 \%)$ & Reference & $142(39.1 \%)$ & Reference \\
\hline \multicolumn{5}{|l|}{ NBCSP Rating Score } \\
\hline Unsatisfactory & $35(10.9 \%)$ & $1.1(0.6-2.0)$ & $25(6.9 \%)$ & $0.7(0.4-1.2)$ \\
\hline Meets expectations & $94(29.3 \%)$ & Reference & $98(27.0 \%)$ & Reference \\
\hline Significantly improves care & $192(59.8 \%)$ & $1.2(0.8-1.7)$ & $240(66.1 \%)$ & $1.6(1.1-2.2)$ \\
\hline \multicolumn{5}{|l|}{ PIP payment } \\
\hline Would make no difference & $221(69.5 \%)$ & $0.6(0.4-0.9)$ & $269(74.1 \%)$ & $1.2(0.8-1.8)$ \\
\hline Neutral & $73(23.0 \%)$ & Reference & $66(18.2 \%)$ & Reference \\
\hline Would make a difference & $24(7.6 \%)$ & $0.5(0.3-1.1)$ & $28(7.7 \%)$ & $0.9(0.5-1.8)$ \\
\hline \multicolumn{5}{|l|}{ External education } \\
\hline Yes & $270(82.3 \%)$ & Reference & $302(83.2 \%)$ & Reference \\
\hline No & $31(9.5 \%)$ & $0.7(0.4-1.1)$ & $33(9.1 \%)$ & $0.6(0.3-0.9)$ \\
\hline I don't remember & $27(8.2 \%)$ & $1.2(0.7-2.1)$ & $28(7.7 \%)$ & $0.8(0.5-1.4)$ \\
\hline
\end{tabular}

*, Full knowledge score is those respondents who scored 9 (Supplementary Data 2); **, Active quality improvement directed to bowel cancer screening is those respondents who scored 7 to 9 (Supplementary Data 2)

\section{Discussion}

Our study identified contextual factors that influenced active QI-CRC practice, which indirectly influenced frequency of FOBT recommendations, but did not appear to influence CRC screening knowledge. Most notably, rating the NBCSP as supporting preventive care, attending external education activities and having sufficient resources to implement QI practice (general) were the contextual factors most strongly associated with QI-CRC practice.

We believe that this is the first quantitative evaluation of CRC screening practice in Australian general practice, since the NBCSP was implemented in 2006. While studies suggest that GP knowledge of screening guidelines may influence population-based screening (Dawson et al., 2017), our findings highlight that FOBT screening recommendations are more varied and complex than the underlying CRC screening knowledge would suggest. Indeed, 'QI practice' may have a greater influence on the frequency of FOBT recommendations than 'knowledge', which does not appear to be as strongly influenced by the contextual factors explored.

Practitioner perception, understanding and support of an organised population-based screening program may influence its success (Aubin-Auger et al., 2011; Dawson et al., 2017; Dimova et al., 2018), even when the program operates independently of general practice (Grogan and Olver, 2014). Few improvements in CRC screening participation in practice have been reported (Dodd et al., 
2018), despite evidence describing effective interventions to enhance screening participation in primary care (Emery et al., 2014). This study begins to identify moderating contextual factors, which little is known (Davis et al., 2018), that potentially account for the variation in practitioner screening knowledge and QI-CRC practice that appear to indirectly influence FOBT screening recommendations in general practice (Perin et al., 2015; Dawson et al., 2017; Şahin and Aker, 2017). The multi-level (patient, organisation and environmental) complexity framework applied is a unique approach to exploring potential contextual predictors of FOBT recommendation, via knowledge and practice improvements. Our study suggests that environmental-level strategies beyond the clinical practice setting (e.g. federal support of general practice, NBCSP enhancements) may enhance the family physician/ GP role in encouraging patients to participate in CRC screening. Such a finding also overcomes the potential implementation barriers frequently encountered in primary care (Moore et al., 2015; Lau et al., 2016).

Better support for QI practice opportunities particularly for those under-resourced practices, such as the NBCSP engaging directly with general practice for example, through the National Cancer Screening Register (Australian Government Department of Health) and Quality Improvement Incentive - Practice Incentives Program (Australian Government Department of Human Services) might facilitate the integration of existing clinical practice with NBCSP activity. QI practice is a fundamental element of quality/preventive care and tends to be more effective in achieving change in routine clinical practice (Grol and Grimshaw, 2003; Holden et al., 2020) and would potentially enhance the practitioner role in the NBCSP without altering the program design.

GPs were not involved in the initial design of the Australian NBCSP program (Grogan and Olver, 2014), which may be reflected in some 'unsatisfactory' NBSCP rating scores in our study (Tong et al., 2004). While efforts are now being made in Australia to better engage the primary health care community (National Bowel Cancer Screening Program), an integrated approach is needed to enhance the fundamental general practice role in preventive and quality care aligned to population-based CRC screening. While individual and practice attributes might indirectly influence the effectiveness of primary care CRC screening interventions, focusing support on high-level systematic QI practice may indirectly improve FOBT screening participation, a hypothesis that warrants further investigation.

Study limitations include the opportunistic email recruitment of a difficult group to recruit (Vaisson $G$ et al., 2018), resulting in a diverse but not necessarily representative sample of practitioners despite measures to maximise the response in the invitation and survey design (Petrovčič et al., 2016). However, respondents are comparable to responders to other similar Australian surveys (Britt et al., 2015) and the sample population is relatively heterogeneous, with most variables showing a diverse response spread allowing comparisons within the cohort. While we found small absolute differences in knowledge scores, the dataset is sufficiently robust to identify step-wise trends and significant associations with contextual factors explored in this study. Such a large and variable sample from every state and territory, has permitted us to canvass a broad range of practice and perspectives being indicative of CRC screening in routine practice.

The study also demonstrates the transferability of survey questions used in other regions (US and UK (Damery et al., 2010)) and modified for the Australian context and its suitability for repeated use. Although the survey methodology does not allow in-depth analysis or association direction (Moore et al., 2015), including the evaluation of targeted general practice interventions and ongoing approval rating of organised CRC screening programs may provide organised screening programs with an indirect indicator of FOBT screening recommendations in general practice.

Our quantitative study has addressed the gap in understanding of contextual factors that indirectly influence CRC screening recommendations in general practice. The findings are most relevant to populationbased CRC screening programs where general practice has an influential role in supporting program delivery and recommending CRC screening participation, particularly for non-adherent or under-screened patients.

\section{Acknowledgments}

The authors wish to acknowledge the Cancer Council SA's Beat Cancer Project for the financial support of this project. The authors also acknowledge the support of the Adelaide Primary Health Network (PHN) and Country SA PHN in pilot testing the online survey. We also acknowledge the in-kind contribution of Healthed Pty Ltd and its staff in the recruitment of survey respondents through its database of GPs. This project was undertaken as part of the No Australians Dying of Bowel Cancer Initiative (NADBCI): the NADBCI wishes to acknowledge the MRFF and Commonwealth Department of Health for the financial support for the broader project aim to eradicate bowel cancer death in Australia.

\section{Conflict of interest disclosure}

Authors OF, ML,JC, DT, DR, RLR and CLM have nothing to disclose. $\mathrm{CAH}$ and IO report grants from Cancer Council SA Beat Cancer Translational Research Scheme, during the conduct of the study. RM is CEO of Healthed which receives funding from multiple sources, primarily the pharmaceutical industry, however no funds were received directly or indirectly for this study.

\section{References}

American Association for Public Opinion Research A (2016). Standard definitions: Final dispositions of case codes and outcome rates for surveys.

Arroyave AM, Penaranda EK, Lewis CL (2011). Organizational change: a way to increase colon, breast and cervical cancer screening in primary care practices. J Community Health, 36, 281-8.

Aubin-Auger I, Mercier A, Lebeau J-P, et al (2011). Obstacles to colorectal screening in general practice: a qualitative study Asian Pacific Journal of Cancer Prevention, Vol 212105 
of GPs and patients. Fam Pract, 28, 670-6.

Australian Bureau of Statistics (ABS) (2016). 'Socio-Economic Indexes for Areas (SEIFA).' Available at http://www.abs. gov.au/ausstats/abs@.nsf/mf/2033.0.55.001 [Accessed 15 July 2019].

Australian Bureau of Statistics (ABS) (2018). The Australian Stastical Geography Standard (ASGS) Remoteness Structure.' Available at http://www.abs.gov.au/websitedbs/ D3310114.nsf/home/remoteness + structure [Accessed 15 July 2019].

Australian Government Department of Health 'National Cancer Screening Register.' Available at https://www.ncsr.gov.au/ [Accessed 3 September 2019].

Australian Government Department of Human Services 'Quality Improvement Incentive.'Available at https://www. humanservices.gov.au/organisations/health-professionals/ services/medicare/practice-incentives-program/guidelines/ quality-improvement-incentive [Accessed 2 September 2019].

Australian Institute of Health and Welfare (2019). National Bowel Cancer Screening Program: monitoring report 2019. Canberra. Available at https://www.aihw.gov.au/ reports/cancer-screening/national-bowel-cancer-screeningprogram-monitoring/contents/table-of-contents [Accessed 3 December 2019].

Britt H, Miller G, Henderson J et al. (2015). General practice activity in Australia 2014-15. Sydney University Press, Sydney.

Cancer Council Australia Colorectal Cancer Guidelines Working Party (2017). 'Clinical practice guidelines for the prevention, early detection and managment of colorectal cancer.' Available at https://wiki.cancer.org.au/australia/ Guidelines:Colorectal_cancer [Accessed 12 February 2020].

Cole S, Young G, Byrne D, Guy J, Morcom J (2002). Participation in screening for colorectal cancer based on a faecal occult blood test is improved by endorsement by the primary care practitioner. J Med Screen, 9, 147-52.

Commonwealth Department of Health GP Workforce Statistics - 2001-02 to 2016-17. Available at http://www.health. gov.au/internet/main/publishing.nsf/Content/General\%20 Practice\%20Statistics-1 [Accessed 15 February 2019].

Damery S, Clifford S, Wilson S (2010). Colorectal cancer screening using the faecal occult blood test (FOBt): a survey of GP attitudes and practices in the UK. BMC Fam Pract, 11, 20.

Davis MM, Freeman M, Shannon J, et al (2018). A systematic review of clinic and community intervention to increase fecal testing for colorectal cancer in rural and low-income populations in the United States - How, what and when?. BMC Cancer, 18, 40.

Dawson G, Crane M, Lyons C, et al (2017). General practitioners' perceptions of population based bowel screening and their influence on practice: a qualitative study. BMC Fam Pract, 18.

Dimova R, Stoyanova R, Tarnovska M (2018). Involvement of general practitioners in colorectal cancer voluntary screening campaign: a mixed-methods study. J BUON, 23, 941-9.

Dodd N, Mansfield E, Carey M, Oldmeadow C, Sanson-Fisher R (2018). Have we increased our efforts to identify strategies which encourage colorectal cancer screening in primary care patients? A review of research outputs over time. Prev Med Rep, 11, 100-4.

Emery JD, Shaw K, Williams B, et al (2014). The role of primary care in early detection and follow-up of cancer. Nat Rev Clin Oncol, 11, 38-48.

Flitcroft KL, Salkeld GP, Gillespie JA, Trevena LJ, Irwig LM (2010). Fifteen years of bowel cancer screening policy in
Australia: putting evidence into practice?. Med J Aust, 193, $37-42$.

Grogan PB, Olver IN (2014). A bowel cancer screening plan at last. Med J Aust, 201, 435-6.

Grol R, Grimshaw J (2003). From best evidence to best practice: effective implementation of change in patients' care. Lancet, 362, 1225-30.

Harris PA, Taylor R, Thielke R, et al (2009). Research electronic data capture (REDCap) - A metadata-driven methodology and workflow process for providing translational research informatics support. J Biomed Inform, 42, 377-81.

Holden CA, Frank O, Caruso J, et al (2020). From participation to diagnostic assessment: a systematic scoping review of the role of primary health care in the National Bowel Cancer Screening Program. Aust J Prim Health, In Press.

Lau R, Stevenson F, Ong BN et al (2016). Achieving change in primary care - causes of the evidence to practice gap: systematic reviews of reviews. Implement Sci, 11, 40.

Mauro M, Rotundo G, Giancotti M (2019). Effect of financial incentives on breast, cervical and colorectal cancer screening delivery rates: Results from a systematic literature review. Health Policy, 123, 1210-20.

Moore G, Audrey S, Barker M, et al (2015). Process evaluation of complex interventions: Medical Research Council guidance. $B M J, 350, \mathrm{~h} 1258$.

National Bowel Cancer Screening Program NBCSP Primary Health Care Engagement Strategy (2016-2020). Australian Government Department of Health Available at http://www. cancerscreening.gov.au/internet/screening/publishing.nsf/ Content/program-frameworks-and-strategies [Accessed 15 February 2019].

National Bowel Cancer Screening Program (2016). National Bowel Cancer Screening Program, Quality Framework. Australian Government Department of Health No. Version 1.0 Available at http://www.cancerscreening.gov.au/internet/ screening/publishing.nsf/Content/program-frameworks-andstrategies [Accessed 3 December 2019].

National Cancer Institute (NCI) National Cancer Institute Primary Care Survey 1999-2000. Available at https:// healthcaredelivery.cancer.gov/crc_surveys/ [Accessed 25 July 2018].

Navarro M, Nicolas A, Ferrandez A, Lanas A (2017). Colorectal cancer population screening programs worldwide in 2016: An update. World J Gastroenterol, 23, 3632-42.

Perin DMP, Saraiya M, Thompson TD, et al (2015). Providers' knowledge, attitudes, and practices related to colorectal cancer control in Brazil. Prev Med, 81, 373-9.

Petrovčič A, Petrič G, Lozar Manfreda K (2016). The effect of email invitation elements on response rate in a web survey within an online community. Comput Hum Behav, 56, 320-9.

Şahin MK, Aker S (2017). Family physicians' knowledge, attitudes, and practices toward colorectal cancer screening. J Cancer Educ, 32, 908-13.

The Royal Australian College of General Practitioners (RACGP) (2016). 'Guidelines for preventive activities in general practice 9th edition.' Available at https://www.racgp.org.au/ your-practice/guidelines/redbook/ [Accessed Sept, 2018].

Tong S, Hughes K, Oldenburg B, Del Mar C (2004). Would general practitioners support a population-based colorectal cancer screening programme of faecal-occult blood testing? Intern Med J, 34, 532-8.

Vaisson G, Witteman HO, Bouck Z, et al (2018). Testing behavior change techniques to encourage primary care physicians to access cancer screening audit and feedback reports: Protocol for a Factorial Randomized Experiment of Email Content, 7, e11.

Vangeest JB, Johnson TP, Welch VL (2007). Methodologies for 
improving response rates in surveys of physicians. Evaluat Health Prof, 30, 303-21.

Youl PH, Jackson C, Oldenburg B, et al (2006). Attitudes, knowledge and practice of CRC screening among GPs in Queensland. Aust Fam Physician, 35, 547-50.

Zajac I, Whibley A, Cole S, et al (2010). Endorsement by the primary care practitioner consistently improves participation in screening for colorectal cancer: a longitudinal analysis. J Med Screen, 17, 19-24.

\section{(ब) (1) 8}

This work is licensed under a Creative Commons AttributionNon Commercial 4.0 International License. 